\title{
Unusual Brain MRI Pattern in 2 Patients with COVID-19 Acute Respiratory Distress Syndrome
}

\author{
(D) S. Toledano-Massiah, (DN. Badat, (D) A. Leberre, (D). Bruel, (D)A. Ray, (D) S. Gerber, (D) M. Zins, and (D). Hodel
}

\section{ABSTRACT}

SUMMARY: We report the cases of 2 patients hospitalized in our intensive care unit with confirmed coronavirus disease 2019 infection in whom brain MR imaging showed an unusual DWI pattern with nodular and ring-shaped lesions involving the periventricular and deep white matter. We discuss the possible reasons for these findings and their relationship to the infection.

ABBREVIATIONS: COVID-19 = coronavirus disease 2019; SARS-CoV-2 = Severe Acute Respiratory Syndrome coronavirus disease 2

$\mathrm{S}$ evere Acute Respiratory Syndrome coronavirus 2 (SARS$\mathrm{CoV}-2)$ is responsible for the coronavirus disease 2019 (COVID-19) pandemic. Although the most common and important presentation of SARS-CoV-2 is respiratory disease, reports of associated neurologic complications are increasing. ${ }^{1}$ A wide spectrum of neurologic symptoms has been described, ranging from nonspecific symptoms such as headache to encephalitis and cerebrovascular disease, either due to direct viral infection or as secondary complications of SARS-CoV2. ${ }^{1}$ This brief report presents 2 patients in our intensive care unit with confirmed COVID-19 infection, in whom brain MR imaging showed an unusual DWI pattern with nodular and ring-shaped lesions of the white matter.

\section{Case Reports}

We report 2 cases of patients admitted to our hospital because of Acute Respiratory Distress Syndrome due to COVID-19. For both of them, CT revealed an interstitial pneumonia, and realtime polymerase chain reaction for SARS-CoV-2 was positive. Endotracheal intubation and mechanical ventilation were required because of severe respiratory failure. Patient 1 was a 52year-old man with a medical history of hypertension, and patient 2, a 44-year-old man with a medical history of diabetes.

Received June 9, 2020; accepted after revision August 3.

From the Departments of Radiology (S.T.-M., N.B., A.L., S.G., M.Z., J.H.), Intensive Care (C.B.), and Neurology (A.R.), Fondation Hôpital Saint-Joseph, Paris, France.

Please address correspondence to Sarah Toledano-Massiah, MD, Fondation Hôpital Saint-Joseph, Department of Radiology, 185 Rue Raymond Losserand, 75014 Paris, France; e-mail: stoledano@hpsj.fr

-- Indicates open access to non-subscribers at www.ajnr.org

http://dx.doi.org/10.3174/ajnr.A6817
During their stay in intensive care unit, both patients presented with sepsis secondary to ventilated-associated pneumonia. Both presented with delayed recovery of consciousness after prolonged sedation. Brain MR imaging was performed at day 31 from intensive care unit hospitalization for patient 1 (day 12 after the interruption of the sedation) and at day 22 for patient 2 (day 10 after the interruption of the sedation).

In both cases, brain MR imaging revealed an unusual pattern, with nodular and ring-shaped lesions involving the periventricular and deep white matter, hyperintense on DWI and FLAIR (Fig 1). The corpus callosum was also involved. There was no involvement of the cortical-subcortical regions. Ring-shaped diffusion restriction was observed in some lesions without postcontrast enhancement. The lesions were larger on FLAIR images than they were on DWI, corresponding to surrounding vasogenic edema. Of note, subtle hemorrhage was also present in some of the lesions in patient 1 (Fig 2). There was no intracranial or supra-aortic trunk vessel obstruction or stenosis on TOF-MRA, or on CT angiography of the supra-aortic vessels.

The CSF real-time polymerase chain reaction for SARS-CoV2 was negative for both patients. Both patients presented with progressive improvement of their neurologic state.

\section{DISCUSSION}

The mechanism of action for SARS-CoV-2 neurologic invasion could be multiple: direct invasion, blood circulation pathway, neuronal pathway, hypoxia injury, immune injury, or related to cytokine storm syndromes and angiotensin-converting enzyme 2 receptor expression. ${ }^{2,3}$

The occurrence of acute stroke in patients with COVID-19 (as suggested in our cases by diffusion restriction) has been previously reported, suggesting either an excessive systemic proinflammatory response in COVID-19 causing dysregulation of 


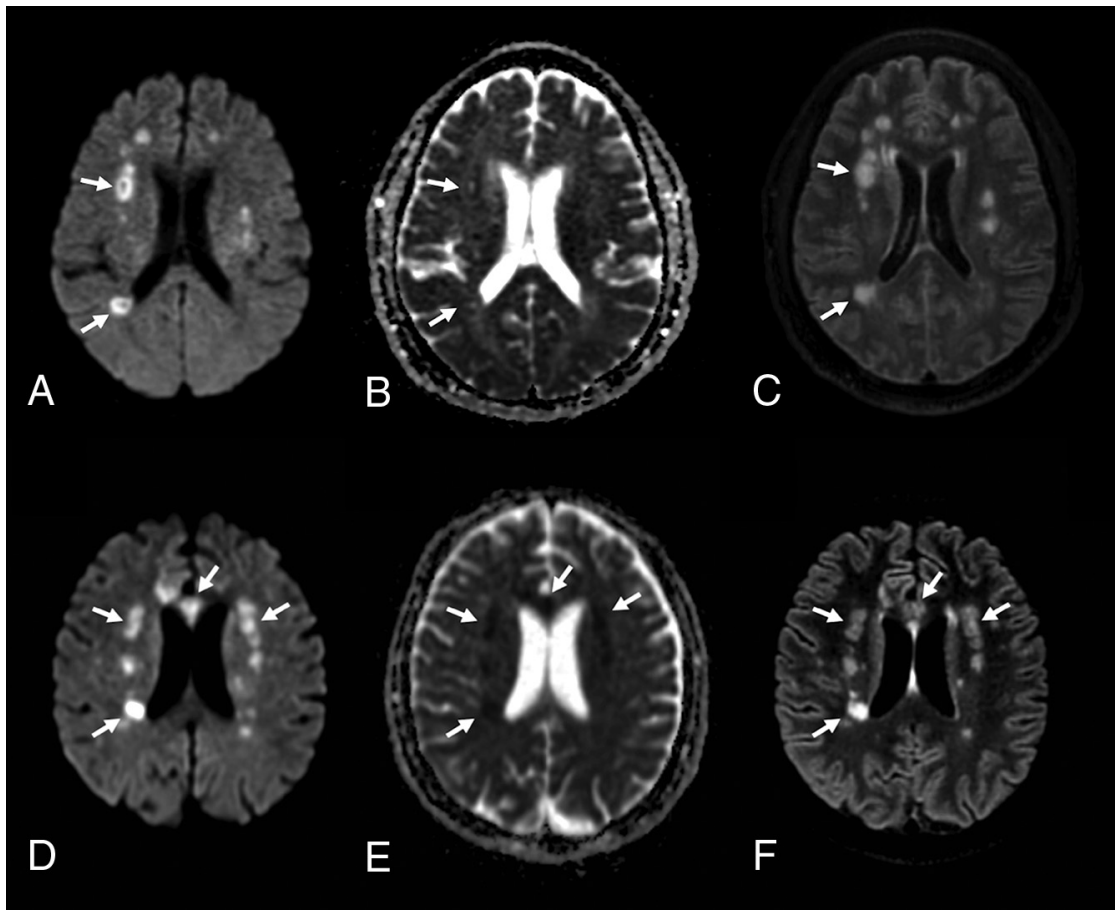

FIG 1. Brain MR imaging in 2 critically ill patients with COVID-19 with delayed recovery of consciousness after prolonged sedation, including a 52-year-old man $(A-C)$, and a 44-year-old man ( $D$ F). Axial diffusion-weighted ( $A$ and $D)$, apparent diffusion coefficient ( $B$ and $E)$, and 3D-FLAIR $(C$ and $F$ ) images in both patients demonstrate nodular and ring-shaped lesions (arrows), involving the periventricular and deep white matter.

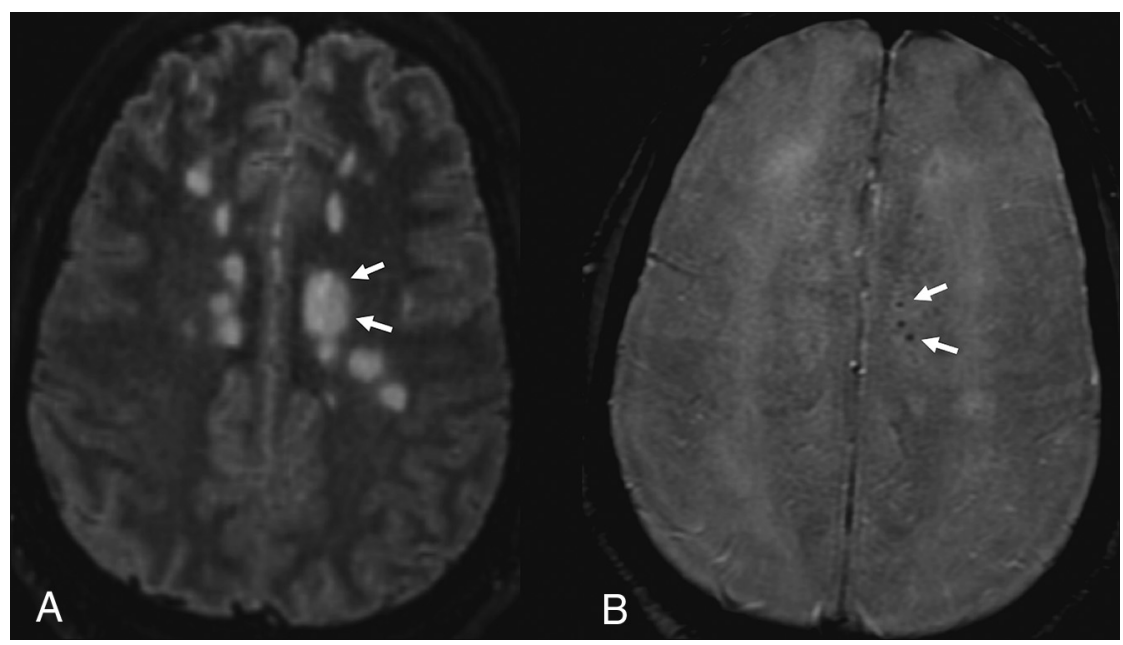

FIG 2. 3D FLAIR image $(A)$ and susceptibility-weighted image $(B)$ show mild hemorrhage within some of the lesions in patient 1 (arrows).

homeostasis with coagulopathy or cardioembolism from virusrelated cardiac injury. ${ }^{4,5}$

However, in our case series, brain MR imaging appeared unusual considering the nodular ring shape as well as the topography (ie, asymmetric involvement of corpus callosum, deep and periventricular white matter) of the lesions. Indeed, such imaging findings could have potentially suggested inflammatory lesions such as acute disseminated encephalomyelitis, which is thought to occur from cross-reactivity in immunity to viral antigens, triggering an autoimmune attack on the CNS.

One patient showed mild punctuated magnetic susceptibility artifacts within some of the brain lesions, suggesting hemorrhage. This could be due to necrosis or microthrombosis, which have both been described in SARS$\mathrm{CoV}-2$, in the brain or in the lung, and could be an additional argument for stroke lesions. Hemorrhagic lesions are also seen in acute necrotizing encephalopathy, which has been described in 1 patient with COVID-19. ${ }^{6}$ Furthermore, the association of microhemorrhages with stroke lesions is suggestive of vasculitis, which has been histologically proved in several other organs including the lung, liver, kidney, or skin, in patients diagnosed with COVID-19. ${ }^{7}$

In conclusion, the etiology and physiopathology of these unusual brain lesions are still not clarified. Neurologists and neuroradiologists should be familiar with the broad spectrum of neuroimaging patterns associated with COVID-19.

\section{REFERENCES}

1. Mao L, Jin H, Wang M, et al. Neurologic manifestations of hospitalized patients with coronavirus disease 2019 in Wuhan, China. JAMA Neurol 2020;77:1-9 CrossRef Medline

2. Baig AM, Khaleeq A, Ali $U$, et al. Evidence of the COVID-19 virus targeting the CNS: tissue distribution, hostvirus interaction, and proposed neurotropic mechanisms. ACS Chem Neurosci 2020;11:995-98 CrossRef Medline

3. Gu J, Gong E, Zhang B, et al. Multiple organ infection and the pathogenesis of SARS. J Exp Med 2005;202:415-24 CrossRef Medline

4. Madjid M, Safavi-Naeini P, Solomon SD, et al. Potential effects of coronaviruses on the cardiovascular system: a review. JAMA Cardiol 2020 Mar 27. [Epub ahead of print] CrossRef Medline

5. Wang D, Hu B, Hu C, et al. Clinical characteristics of $\mathbf{1 3 8}$ hospitalized patients with 2019 novel coronavirus-infected pneumonia in Wuhan, China. JAMA 2020 Feb 7. [Epub ahead of print] CrossRef Medline

6. Poyiadji N, Shahin G, Noujaim D, et al. COVID-19-associated acute hemorrhagic necrotizing encephalopathy: CT and MRI features. Radiology 2020;296:E119-20 CrossRef Medline

7. Xu Z, Shi L, Wang Y, et al. Pathological findings of COVID-19 associated with acute respiratory distress syndrome. Lancet Respir Med 2020;8:420-42 CrossRef Medline 